\title{
Dichloroacetate restores drug sensitivity in paclitaxel-resistant cells by inducing citric acid accumulation
}

\author{
Xiang Zhou ${ }^{1 \dagger}$, Ruohua Chen ${ }^{1 \dagger}$, Zhenhai $\mathrm{Yu}^{2}$, Rui Li ${ }^{1}$, Jiajin Li ${ }^{1}$, Xiaoping Zhao ${ }^{1}$, Shaoli Song ${ }^{1}$, Jianjun Liư ${ }^{*}$ \\ and Gang Huang ${ }^{1,3^{*}}$
}

\begin{abstract}
Background: The Warburg effect describes the increased reliance of tumor cells on glycolysis for ATP generation. Mitochondrial respiratory defect is thought to be an important factor leading to the Warburg effect in some types of tumor cells. Consequently, there is growing interest in developing anti-cancer drugs that target mitochondria. One example is dichloroacetate (DCA) that stimulates mitochondria through inhibition of pyruvate dehydrogenase kinase.

Methods: We investigated the anti-cancer activity of DCA using biochemical and isotopic tracing methods.

Results: We observed that paclitaxel-resistant cells contained decreased levels of citric acid and sustained mitochondrial respiratory defect. DCA specifically acted on cells with mitochondrial respiratory defect to reverse paclitaxel resistance. DCA could not effectively activate oxidative respiration in drug-resistant cells, but induced higher levels of citrate accumulation, which led to inhibition of glycolysis and inactivation of P-glycoprotein.

Conclusions: The abilityof DCA to target cells with mitochondrial respiratory defect and restore paclitaxel sensitivity by inducing citrate accumulation supports further preclinical development.
\end{abstract}

Keywords: Dichloroacetate, Paclitaxel, Mitochondrial respiratory defect, Pyruvate dehydrogenase kinase, Warburg effect

\section{Background}

More than 80 years ago, Warburg first observed that glycolysis is increased in cancer cells that depend largely on this metabolic pathway to meet their energy needs [1]. Positron emission tomography (PET) has since confirmed that most tumors undergo increased glucose uptake and metabolism [2]. This phenomenon is known as the Warburg effect, which is believed to reflect mitochondrial injury and alternative isoforms of glycolytic enzymes in cancer cells $[3,4]$. Mitochondrial respiratory injury is due primarily to mutations in mitochondrial DNA, reduced activity of key

\footnotetext{
*Correspondence: ljjsh@133sh.com; huang2802@163.com

${ }^{\dagger}$ Equal contributors

'Department of Nuclear Medicine, Ren Ji Hospital, School of Medicine, Shanghai Jiao Tong University, 1630 Dongfang Road, Shanghai 200127, China

${ }^{3}$ Department of Cancer Metabolism, Institute of Health Sciences, Chinese Academy of Sciences and Shanghai Jiao Tong University School Medicine, Shanghai, China

Full list of author information is available at the end of the article
}

tricarboxylic acid (TCA) cycle enzymes, and a hypoxic microenvironment. Tumor cells with mitochondrial respiratory injury have decreased TCA cycle and/or ETC. flux, which can lead to reduced production of $\mathrm{CO}_{2}$ and ATP with decreased $\mathrm{O}_{2}$ consumption [5-7]. As a result, targeting mitochondria in cancer therapy is receiving increased attention.

Dichloroacetate (DCA) activates pyruvate dehydrogenase complex (PDC) by inhibiting pyruvate dehydrogenase kinase (PDK). PDC is an important gateway enzyme that influences glycolysis and glucose oxidation [8]. Activation of PDC converts pyruvate into acetyl-CoA, which is converted into citric acid in the mitochondria, stimulating mitochondrial oxidative phosphorylation [9].

DCA induces apoptosis, decreases proliferation, and inhibits tumor growth through inhibition of PDK [10]. DCA has no apparent toxicity except for peripheral neuropathy, which is reversible upon drug discontinuation [11]. Recently, DCA has been used increasingly in research on 
prostate, breast and colorectal cancer [12-14]. DCA toxicity is specific to the tumor type [15-17], and tumor cells with mitochondrial injury are sensitive to DCA through an unknown mechanism [18].

Paclitaxel is an important clinical agent for the treatment of malignant breast, prostate and non-small lung tumors, but resistance is a serious problem $[19,20]$. Contributing to drug resistance is the high expression of anti-apoptotic proteins as well as the high expression of proteins that lets cancer evade cancer treatment [21], and finding a way to reverse multidrug resistance is a major target of cancer research.

A549/Taxol cells are paclitaxel resistant and exhibit high levels of P-glycoprotein expression [22]. In this study, we observed that DCA targets A549/Taxol cells to enhance the sensitivity to paclitaxel. Given that DCA is known to target cells with mitochondrial respiratory defects [18], we investigated whether the mitochondria of A549/Taxol cells were damaged. DCA activates PDC to stimulate mitochondria, therefore we assessed whether DCA specifically targets drug-resistant cells because of its restoration of mitochondrial function.

We observed that the paclitaxel resistance in lung cancer cells was closely correlated with mitochondrial injury. DCA could not activate oxidative respiration in drug-resistant cells but did inhibit glucose uptake significantly. These results have improved our understanding of the mechanism by which DCA affects tumor cell suppression, mitochondrial function, glucose metabolism and drug resistance.

\section{Materials and methods}

\section{Chemicals and reagents}

DCA, 3-bromopyruvate, sodium pyruvate, coenzyme A, glutamine, uridine and daunomycin were purchased from Sigma. Dulbecco's Modified Eagle's Medium (DMEM) and fetal bovine serum (FBS) were purchased from GIBCO. ${ }^{14} \mathrm{C}$-6-glucose was purchased from Moravek Biochemicals. Mouse monoclonal anti-P-glycoprotein was purchased from Santa Cruz Biotechnology and rabbit polyclonal antiPDK2 was purchased from Protein Tech. ${ }^{99 \mathrm{~m}}$ Tc-MIBI (methoxyisobutylisonitrile) was purchased from Shanghai Hinko and ${ }^{18} \mathrm{~F}$-fluorodeoxyglucose was produced in our laboratory.

\section{Cell culture and transient transfection}

The human lung adenocarcinoma cell lines A549and H1299wereobtained from the Chinese Academy of Sciences. HCCLM3 cell line [23] (human HCC cell lines with high metastatic potentials established at the Liver Cancer Institute, Zhong Shan Hospital, Fudan University, China) was also used in this study. The paclitaxel-resistant A549/Taxol cell line was developed as previously described [22,24]. Briefly, the parental A549 cell line was exposed to an initial paclitaxel concentration of $1 \mu \mathrm{g} / \mathrm{L}$. The concentration of paclitaxel was escalated stepwise to $1 \mu \mathrm{g} / \mathrm{mL}$ with changes of medium biweekly. The surviving resistant cells were renamed A549/Taxol and cultured in DMEM containing $5 \mathrm{mM}$ glucose supplemented with $10 \% \mathrm{FBS}$, sodium pyruvate and l-glutamine. The mitochondrially defective clone, A549/MD cells, was produced as previously described [25]. Briefly, the cells were produced by exposure to $5 \mathrm{ng} / \mathrm{ml}$ ethidium bromide, which was increased in a stepwise fashion to a limit of $50 \mathrm{ng} / \mathrm{ml}$. A549/MD cells were maintained in DMEM supplemented with 10\% FBS, $1 \mathrm{mM}$ sodium pyruvate, $50 \mathrm{mM}$ uridine, $5 \mathrm{mM}$ glucose and $2 \mathrm{mM}$ l-glutamine. Hypoxic treatment was performed in a specially designed hypoxia incubator (Chermo Electron) with $1.5 \% \mathrm{O}_{2}, 5 \% \mathrm{CO}_{2}$ and $93.5 \% \mathrm{~N}_{2}$. Lipofectamine 2000 (Invitrogen) was used for transient transfection. siRNAPDK2 and control was purchased from Pharma China.

\section{Measurement of intracellular ATP}

Relative cellular ATP content was measured using the ATP bioluminescent somatic cell assay kit (Sigma). Cell lysates were collected, and luminescence was measured using a luminescence reader and normalized to the protein concentration.

\section{Mitochondrial membrane potential}

Cells were plated insix-well plates with $2 \times 10^{5}$ cells per well. $5,5^{\prime}, 6,6^{\prime}$-tetrachloro-1,1',3,3' -tetraethylbenzimidazol-carbocyanineiodide (JC-1) was added to the medium at a final concentration of $10 \mu \mathrm{g} / \mathrm{ml}$, and the cells were allowed to stain for30 min. After JC-1 staining, cells were washed twice with PBS and resuspended in $300 \mu \mathrm{l}$ PBS. JC-1 intensity was examined using fluorescence-activated cell sorting. Mitochondrial membrane potential was calculated using the followingformula:JC-1 intensity of Q2 quadrant/JC-1 intensity of Q4 quadrant.

\section{Analysis of enzyme activity}

For analysis of complex I activity from A549, A549/Taxol and A549/MD cells, the Complex I Enzyme Activity Microplate Assay Kit was used (Abcam). Cell lysates were collected and added to each well of the microplate and incubated for $3 \mathrm{~h}$. After rinsing all wells, assay solution was added and absorbance at $450 \mathrm{~nm}$ was measured at $\sim 1$-min intervals for $30 \mathrm{~min}$.

Phosphofructokinase (PFK) activity was measured using the PFK activity colorimetric assay kit (BioVision). A549, A549/Taxol cells were treated with DCA for $24 \mathrm{~h}$. Cell extracts were prepared and assayed and absorbance at $450 \mathrm{~nm}$ was measured at 10,20 and $30 \mathrm{~min}$.

For pyruvate dehydrogenase (PDH) activity measurement, mitochondria from A549 and A549/Taxol cells treated with DCA for $24 \mathrm{~h}$ were isolated using the Mitochondria Isolation Kit for Mammalian Cell (Thermo). After mitochondria were sonicated, PDH activity was 
assayed by measuring the reduction of $\mathrm{NAD}^{+}$at $340 \mathrm{~nm}$ in the presence of $200 \mu \mathrm{M}$ TPP, $40 \mu \mathrm{M}$ coenzyme A, and $4 \mu \mathrm{M}$ pyruvate. The assay was carried out in the presence of $2.5 \mu \mathrm{M}$ rotenone to prevent $\mathrm{NADH}$ consumption by complex I [26].

\section{Oxygen consumption and extracellular acidification rate analysis}

Oxygen consumption rate (OCR) and extracellular acidification rate (ECAR) were measured using the Seahorse XF24 analyzer (Seahorse Bioscience). Cells were seeded in a 24-well cell culture microplate and allowed to attach overnight. Approximately $30 \mathrm{~min}$ prior to the assay, culture medium was changed to the Seahorse assay medium, DMEM containing $5 \mathrm{mM}$ glucose or $2 \mathrm{mM}$ l-glutamine. The waveguides deliver light rays at various excitation wave lengths (oxygen $=532 \mathrm{~nm}, \mathrm{pH}=470 \mathrm{~nm}$ ) and transmit a fluorescent signal (oxygen $=650 \mathrm{~nm}, \mathrm{pH}=530 \mathrm{~nm}$ ). OCR and ECAR were measured, as described previously $[27,28]$.

\section{Metabolite and citrate extraction using GC/MS analysis}

Metabolite and citrate extraction was performed using GC/MS analysis, as described previously [29]. Briefly, cells were cultured in 6 -cm dishes, rinsed with $1 \mathrm{ml}$ ice-

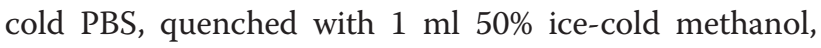
and the extracts were vortexed at $4^{\circ} \mathrm{C}$ for $30 \mathrm{~min}$. After samples were centrifuged and evaporated, tert-butyldimethylsilylderivatization was initiated, and the samples were incubated at $75^{\circ} \mathrm{C}$ for $1 \mathrm{~h}$. GC/MS analysis was performed using the Agilent 6890GC.

\section{${ }^{18}$ F-FDG uptake}

Cells were cultured in 12-well plates, detached, washed twice, and incubated in $500 \mu \mathrm{l}$ DMEM containing $4 \mu \mathrm{Ci} / \mathrm{ml}$

${ }^{18} \mathrm{~F}$-FDG for $1 \mathrm{~h}$ at $37^{\circ} \mathrm{C}$. Pellets were washed twice with ice-cold PBS. Lysates were produced using $500 \mu \mathrm{l} 0.1 \mathrm{M}$ $\mathrm{NaOH}$ and the radioactivity of the whole-cell lysates was assayed using a well gamma-counter [4]. Cell protein content was measured in parallel using the BCA protein assay kit (BIB).

\section{${ }^{14} \mathrm{CO} 2$ release assay}

Cells cultured in 100-mm dishes were placed in an airtight chamber. Five milliliters of DMEM containing ${ }^{14} \mathrm{C}$-6-glucose $(2 \mu \mathrm{Ci} / \mathrm{ml})$ was added and the cells were incubated at $37^{\circ} \mathrm{C}$ for $2 \mathrm{~h}$ with a constant airflow $\left(5 \% \mathrm{CO}_{2}-95 \%\right.$ air, $15 \mathrm{ml} / \mathrm{min}$ ); ${ }^{14} \mathrm{CO}_{2}$ was trapped using $16 \mathrm{ml}$ amine-based absorber. Four milliliters of absorber-trapped ${ }^{14} \mathrm{CO}_{2}$ was transferred to vials containing $12 \mathrm{ml}$ permafluor $\mathrm{E}+$ and radioactivity was counted using a liquid scintillation counter [30]. The cell residue was used to determine protein content.

\section{Analysis of P-glycoprotein activity}

${ }^{99 \mathrm{~m}}$ Tc-methoxyisobutylisonitrile $\left({ }^{99 \mathrm{~m}} \mathrm{Tc}-\mathrm{MIBI}\right)$ is a transport substrate for P-glycoprotein [31]. For quantitative assessment of transporter function in multidrug-resistant cells, ${ }^{99 \mathrm{~m}}$ TC-MIBI was added to cultured tumor cells. Uptake and efflux were determined as described previously [32]. Briefly, the cells were cultured in 12-well plates, and the medium was removed and replaced with $500 \mu \mathrm{l}$ medium containing $4 \mu \mathrm{Ci} / \mathrm{ml}{ }^{99 m} \mathrm{TC}-\mathrm{MIBI}$ and various modulators for 30 or $120 \mathrm{~min}$ at $37^{\circ} \mathrm{C}$. The cells were subsequently lysed in $0.1 \mathrm{mM} \mathrm{NaOH}$. Efflux of ${ }^{99 \mathrm{~m}} \mathrm{TC}-\mathrm{MIBI}$ was performed as described previously [26]. Cells were incubated in DMEM containing ${ }^{99 \mathrm{~m}} \mathrm{TC}-\mathrm{MIBI}$ and various modulators for $1 \mathrm{~h}$, washed twice with $\mathrm{PBS}$, and the medium without ${ }^{99 m}$ TC-MIBI was replaced. The cells were lysed for 10, 30, 60 or 90 min with $\mathrm{NaOH}$. All samples were measured using a well gammacounter. In parallel, cell protein content was measured using the BCA protein assay kit.

Activity of P-glycoprotein was measured by analysis of the intracellular accumulation of doxorubicin, as described previously [33]. The cells were cultured in 12-well plates, containing $10 \mathrm{mg} / \mathrm{L}$ doxorubicin and various modulators for $2 \mathrm{~h}$ at $37^{\circ} \mathrm{C}$. Cells were resuspended in $0.2 \mathrm{ml} \mathrm{PBS}$ and analyzed by flow cytometry with an excitation wavelength of $488 \mathrm{~nm}$ for the mean fluorescence intensity of intracellular doxorubicin.

\section{Cell Counting Kit (CCK)-8 assay}

Cell survival was measured using the CCK-8 assay (Dojindo Molecular Technologies), as described previously [34]. Cells were plated in 96-well plates at 6000 cells/well. After $24 \mathrm{~h}$ culture, the medium was replaced with DMEM containing $10 \%$ FBS and drugs at the indicated concentrations. After incubation for $24 \mathrm{~h}, 10 \mu$ lcholecystokinin- 8 was added to each well, and the cells were incubated for a further $2 \mathrm{~h}$. Absorbance was read at $450 \mathrm{~nm}$ using an enzyme microplate reader. The half-maximal inhibitory concentration $\left(\mathrm{IC}_{50}\right)$ was calculated using GraphPad Prism version 5.0. Resistance index was calculated using the following formula: $\mathrm{IC}_{50}$ resistant cells/ $\mathrm{IC}_{50}$ parental cells.

\section{In vivo effect of DCA and paclitaxel in A549/Taxol cells xenograft}

All animal experiments were performed in accordance with the National Institutes of Health Guide for the Care and Use of Laboratory Animals and were approved by Affiliated RenJi Hospital of Shanghai Jiaotong University. Male 4-6week-old BALB/c athymic (nut/nut) mice (SLAC Laboratory Animals) were subcutaneously inoculated with $5 \times 10^{6}$ A549/Taxol cells in serum-free medium. Mice were randomized into four groups of six 7 days after inoculation: (1) vehicle (control); (2) paclitaxel alone; (3) DCA alone; and (4) DCA combined with paclitaxel. DCA (0.75 g/L) was 
added to drinking water for mice in the DCA alone and DCA + paclitaxel groups. Mice in the paclitaxel alone and $\mathrm{DCA}+$ paclitaxel groups were intraperitoneally injected with $6 \mathrm{mg} / \mathrm{kg}$ paclitaxel, which was repeated once weekly for a total of three doses $(18 \mathrm{mg} / \mathrm{kg})$. Tumor volume was calculated using the following formula: volume $\left(\mathrm{mm}^{3}\right)=$ $(\text { width })^{2} \times$ length $\times 0.5$. Tumor volume and body weight were measured twice weekly. Five weeks after treatment, mice were sacrificed and weighed, and tumors were excised and weighed.

\section{Statistical analysis}

Statistical differences between the groups were assessed using two-tailed analysis of variance and $t$ tests. $P<0.05$ was considered statistically significant.

\section{Results}

Paclitaxel-resistant cells are associated with mitochondrial dysfunction

A549/Taxol cells had a paclitaxel resistance index of 32 (Figure 1A). Paclitaxel increases production of reactive oxygen species (ROS) in tumor cells that may result in mitochondrial injury because of long-term ROS-induced oxidative stress and a compromised mtRNA self-repair mechanism [35].

Electron microscopy showed that mitochondrial volume increased and cristae appeared less wellformed in A549/Taxol cells compared withA549 cells (Figure 1B). Mitochondrial respiratory injury can be evaluated through the oxygen consumption rate (OCR) and release of ${ }^{14} \mathrm{C}-\mathrm{CO}_{2}$. The oxygen consumption rate of the two cell lines was measured with glucose or glutamine (Figure 1C), and A549/Taxol cells showed significantly reduced oxidative capacity with either carbon source. Release of ${ }^{14} \mathrm{C}-\mathrm{CO}_{2}$ was measured to evaluate mitochondrial respiration using ${ }^{14} \mathrm{C}$-6-glucose as a carbon source, and A549/Taxol cells released less ${ }^{14} \mathrm{C}-\mathrm{CO}_{2}$ than A549 cells did (Figure 1D). Mitochondrial membrane potential is an indicator of mitochondrial function in stable cell lines, and damage to the electron transport chain can decrease mitochondrial membrane potential [36]. Lower mitochondrial membrane potential was observed in A549/Taxol cells (Figure 1E), and the activity of complex I was also greatly reduced in these cells compared withA549 cells (Figure 1F). These results demonstrated that the mitochondria of paclitaxelresistant cells were severely damaged.

Mitochondrial damage in tumor cells can lead to metabolic changes. Analysis of RT-PCR data confirmed that the mRNA levels of key enzymes involved in glucose metabolism were increased, and expression of the tumor suppressor gene p53 was decreased in A549/Taxol compared withA549 cells (Figure 1G). Similarly, the protein expression of p53 was decreased in A549/Taxol cells compared withA549 cells (Figure $1 \mathrm{H}$ ).

\section{DCA specifically reverses drug resistance in cells with mitochondrial respiratory deficiency}

Mitochondria are pivotal in energy metabolism and regulation of apoptosis [37], therefore we hypothesized that the artificial/induced mitochondrial respiratory defect in A549 cells may increase paclitaxel resistance. A549/MD cells derived from A549 cells were defective in mitochondrial respiration, and transmission electron microscopy revealed that mitochondria in these cells were abnormally swollen with disorganized cristae (Figure 2A). Biochemical analysis showed that A549/MD cells released less ${ }^{14} \mathrm{C}$ $\mathrm{CO}_{2}$ and had lower minimal complex I activity than A549 cells had (Figure 2B; Additional file 1: Figure S1), indicating a severe deficiency in mitochondrial respiration. The effect of mitochondrial respiratory defects on the cellular response to anti-cancer agents was investigated, and as expected, A549/MD cells possessed a higher resistance to paclitaxel than A549 cells (Figure 2D) with a resistance index of 37.

P-glycoprotein is an important resistance-associated protein that can secrete antitumor drugs, and paclitaxel is a known P-glycoprotein substrate [36]. Expression of Pglycoprotein was increased in the stable A549/MD cell line (Additional file 2: Figure S2), suggesting that it contributes to or is responsible for paclitaxel resistance in A549/MD cells.

Drug resistance profoundly affects the clinical treatment of tumors. Paclitaxel-resistant cells were associated with mitochondrial respiratory defects, therefore preventing drug resistance by targeting mitochondrial respiratory defects and restoring drug sensitivity may be a promising area of research.

DCA is a known regulator of PDK and is thought to act specifically on cells with mitochondrial respiratory defects. In this study, DCA treatment for $48 \mathrm{~h}$ resulted in more A549/MD and A549/Taxol cell death with injured mitochondria than occurred in A549 cells (Figure 2C). In addition, DCA significantly increased the sensitivity of drug-resistant cell lines (both A549/Taxol and A549/MD cells) to paclitaxel (Figure 2D), while the parental A549 cells were unaffected. These results indicate a link between drug resistance and mitochondrial respiratory defects, and reversal of drug resistance by DCA is specific to drugresistant cells exhibiting mitochondrial respiratory defects.

\section{DCA cannot effectively restore mitochondrial respiration in paclitaxel-resistant cells, but inhibits glycolysis significantly more than A549 cells with functioning mitochondria}

Next we assessed whether DCA specifically targets drugresistant cells with mitochondrial respiratory defect by 
A

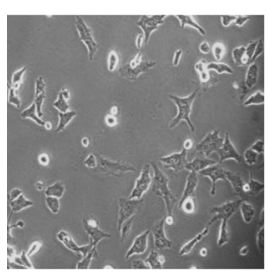

A549

C

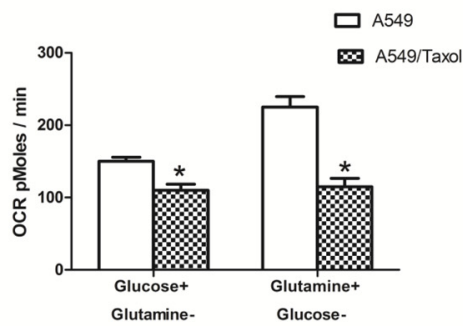

E

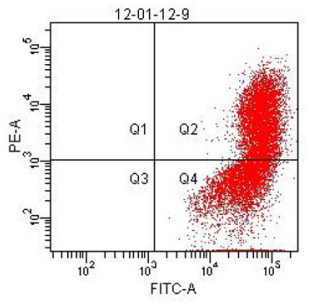

A549

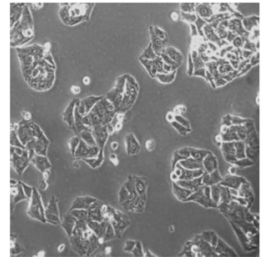

A549/Taxol
A549/Taxol

B

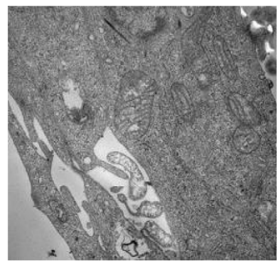

A549

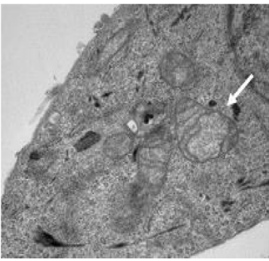

A549/Taxol

D

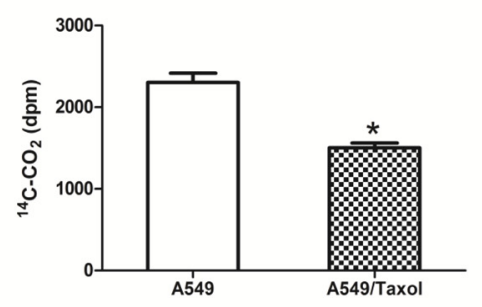

F

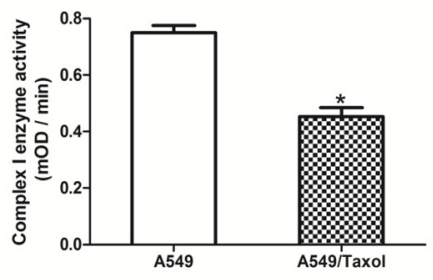

G

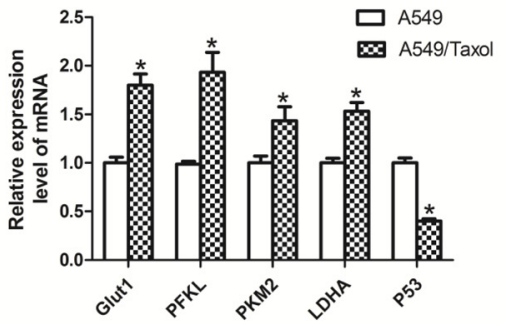

H

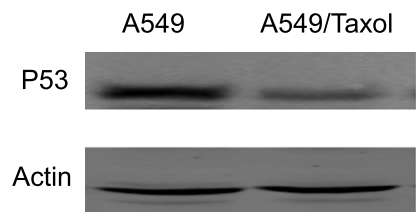

Figure 1 Characterization of alterations in mitochondrial respiration in paclitaxel-sensitive (A549) and paclitaxel-resistant (A549/Taxol) cells. (A) A549 and A549/Taxol cells at 200x magnification. (B) Transmission electron microscopy of mitochondrial morphology. (C) Oxygen consumption. Cells were cultured in DMEM using glucose or glutamine as a carbon source. (D) Release of ${ }^{14} \mathrm{C}-\mathrm{CO}_{2}$. Cells $\left(5 \times 10^{6}\right)$ were cultured in $4 \mathrm{ml}$ low-glucose DMEM containing $0.5 \mu \mathrm{Ci} / \mathrm{ml}^{14} \mathrm{C}-6$-glucose for $2 \mathrm{~h}$. Released ${ }^{14} \mathrm{C}-\mathrm{CO}_{2}$ was measured. (E) Mitochondrial membrane potential was calculated using the following formula: JC-1 intensity of Q2 quadrant / JC-1 intensity of Q4 quadrant. (F) Mitochondrial oxidative phosphorylation complex I enzyme activity. (G) Comparative analysis of the expression of key glycolysis enzymes. (H) p53expression in A549 and A549/Taxol cells by western blot analysis. ${ }^{*} P<0.05$. Data are mean \pm standard error of the mean (SEM) of three independent experiments.

assisting the recovery of mitochondrial function. Glutamine and glucose are important carbon sources for oxidative phosphorylation in tumor cells [29]. DCA increased oxygen consumption by $19 \%$ in A549 cells but only by $9 \%$ in A549/ Taxol cells, with glucose as a carbon source (Figure 3A). This observation was consistent with ${ }^{14} \mathrm{C}-\mathrm{CO}_{2}$ expiration data (Figure 3B). With glutamine as a carbon source, DCA significantly inhibited glutamine oxidation via a mechanism that is not understood. DCA inhibited glutamine oxidation by $34.4 \%$ in A549/Taxol cells and $19.1 \%$ in A549 cells. DCA inhibited glutamine oxidation more effectively in drugresistant cells exhibiting mitochondrial respiratory defects (Figure 3C). These results suggest that the ability of DCA to target specifically tumor cells with mitochondrial respiratory defects is not due to the restoration of mitochondrial function in A549/Taxol cells. 
A

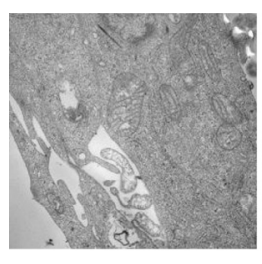

A549

C

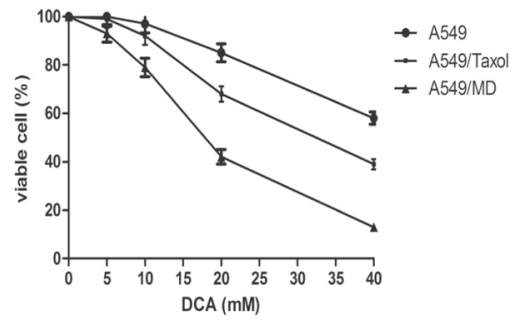

B

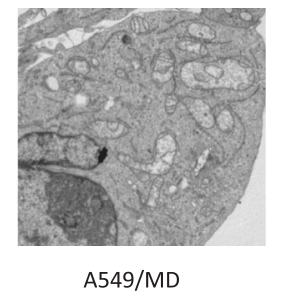

D
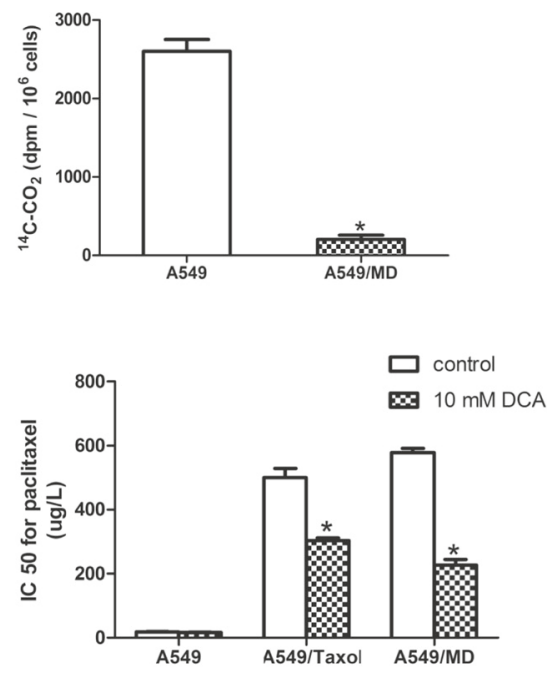

Figure 2 DCA affects paclitaxel resistance in cells with damaged mitochondria. (A) Transmission electron microscopy of mitochondrial morphology in mitochondrial respiration-deficient A549/MD cells. (B) Release of ${ }^{14} \mathrm{C}^{-} \mathrm{CO}_{2}$ from A549/MD cells. (C) Viability of A549, A549/Taxol and A549/MD cells treated with DCA for 48 h. (D) IC $C_{50}$ for paclitaxel in A549, A549/Taxol and A549/MD cells after treatment for $48 \mathrm{~h}$ with 0 and $10 \mathrm{mM}$ DCA. ${ }^{*} P<0.05$. Data are mean \pm SEM of three independent experiments.

Although DCA could not effectively restore mitochondrial respiration in resistant cells, DCA inhibited ECAR significantly compared to control treatment in both A549 and A549/Taxol cells. However, there was a larger difference in ECAR rate between control and DCA treated cells in the A549/Taxol cells than the A549 (Figure 3D). DCA reduced glucose uptake by $35 \%$ in A549/Taxol cells, but only by $12 \%$ in A549 cells (Figure 3E). These results were significant as glycolysis was the main source of ATP in cells with severely injured mitochondria [25]. This result was consistent with specific inhibition of glycolytic ATP generation using 3-bromopyruvate (3BrPA), which is an inhibitor of hexokinase II with an inhibitory effect on glycolysis (Figure 3F). Whether DCA decreased ATP production via inhibition of glycolysis was investigated, and 10 mM DCA for 24 h decreased ATP generation in A549/ Taxol cells, but ATP production was unaffected in A549 cells (Figure 3G).

\section{DCA suppresses P-glycoprotein function in paclitaxel-resistant cells}

Compared with A549 cells, A549/Taxol cells expressed higher levels of P-glycoprotein, which would be expected to increase the excretion of antitumor drugs via active transport. However, this process requires an energy supply. Although DCA had no effect on P-glycoprotein expression (Figure 4A), decreased levels of ATP in DCA-treated drugresistant cells could lead to functional inactivation of P-glycoprotein as seen in Figure 3G, and this was investigated by measuring the uptake and excretion of the
P-glycoprotein radioactive substrate ${ }^{99 m}$ Tc-MIBI [38]. Verapamil was used as a positive control, because it is a P-glycoprotein substrate that inhibits the transport of drugs by this protein. DCA treatment increased ${ }^{99 \mathrm{~m}} \mathrm{Tc}$ MIBI accumulation in A549/Taxol cells by 20\%, but as the parental A549 cell line did not express Pglycoprotein, DCA did not affect ${ }^{99}$ Tc-MIBI uptake (Figure 4B). DCA significantly prolonged the intracellular retention of ${ }^{99 \mathrm{~m}}$ Tc-MIBI (Figure $4 \mathrm{C}$ ).

Doxorubicin is another known substrate of P-glycoprotein [33], and DCA treatment also significantly increased doxorubicin accumulation in A549/Taxol cells but not in A549 cells (Figure 4D). These results suggest that DCA is able to inhibit the function of P-glycoprotein in cells with mitochondrial respiratory defects.

\section{DCA increases citrate accumulation in cells with mitochondrial injury}

The above results indicated that DCA could inactivate Pglycoprotein to enhance sensitivity of A549/Taxol cells to paclitaxel by inhibiting glycolysis. We elucidated why DCA inhibited glycolysis in A549/Taxol cells but did not activate the TCA cycle. DCA targets PDK and increases $\mathrm{PDH}$ activity, thus it would be expected to stimulate the TCA cycle. DCA was found to activate PDC in both A549 and A549/Taxol cells (Figure 5A), but the TCA cycle was not stimulated in either cell type. We therefore investigated whether this resulted in accumulation of TCA cycle substrates in A549/Taxol cells. 

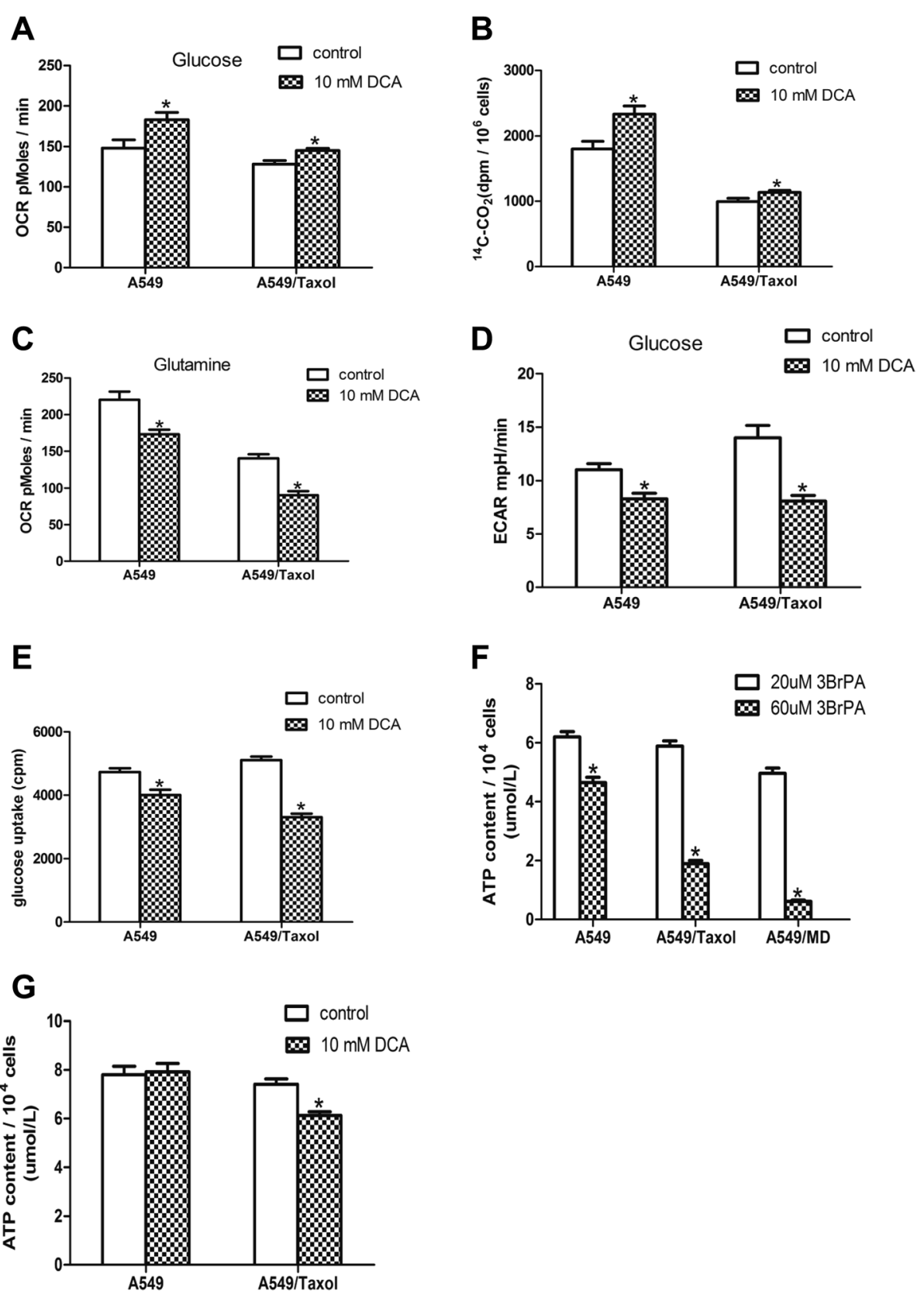

Figure 3 Influence of DCA on mitochondrial respiration and glycolysis. (A) OCR in A549 and A549/Taxol cells treated with 0 or $10 \mathrm{mM} \mathrm{DCA} \mathrm{for} 24 \mathrm{~h}$ with glucose as carbon source. (B) Release of ${ }^{14} \mathrm{C}-\mathrm{CO}_{2}$ by $\mathrm{A} 549$ and A549/Taxol cells treated with 0 or $10 \mathrm{mM}$ DCA for 24 h. (C) OCR in A549 and A549/ Taxol cells treated with 0 or 10 mM DCA for $24 \mathrm{~h}$ with glutamine as carbon source. (D) Influence of DCA on ECAR in A549 and A549/Taxol cells. (E) Influence of DCA on glucose uptake in A549 and A549/Taxol cells incubated in DMEM with 18 F-FDG for $1 \mathrm{~h}$. Uptake of radioactivity was measured with a well gamma counter. (F) Effect of 3BrPA on ATP generation by glycolysis in A549, A549/Taxol and A549/MD cells. (G) Influence of DCA on ATP production after treatment with 0 or $10 \mathrm{mM}$ DCA for $24 \mathrm{~h}$. ${ }^{*} P<0.05$. Data are mean \pm SEM of three independent experiments.

Acetyl-CoA is the product of PDH and an important intermediate in energy generation and fatty acid synthesis. However, acetyl-CoA does not pass through the mitochondrial membrane, and is quickly converted into citric acid by citrate synthase (which also has a high affinity for acetyl-CoA). Citrate is then transported out of the mitochondria via the citric acid shuttle, or enters the TCA cycle [39]. Cells harboring mitochondrial respiratory defects do not possess a normally functioning TCA cycle, therefore citric acid generated in DCAtreated cells cannot be consumed in a timely fashion, and instead accumulates in the cytoplasm. Citrate is an important metabolite that regulates the balance between glycolysis and oxidative phosphorylation in cancer cells. 
A

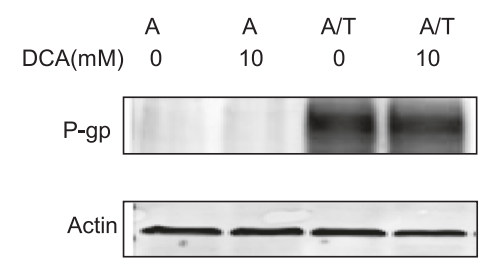

B
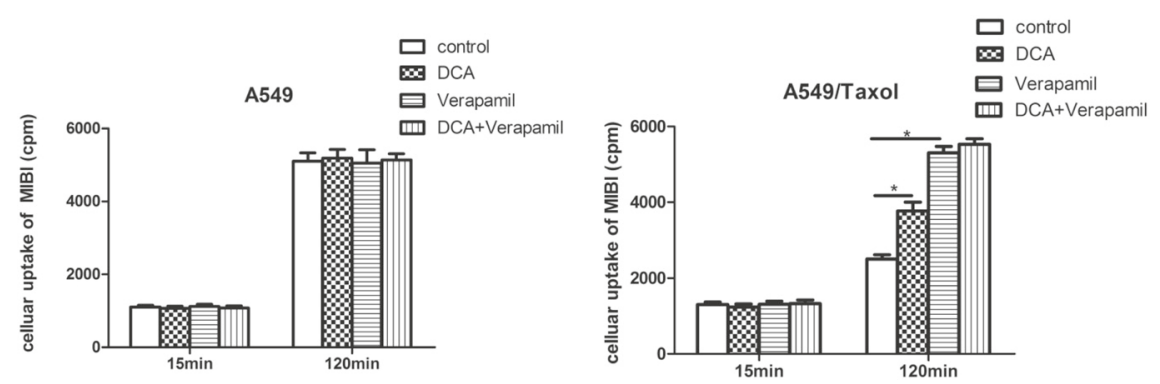

C
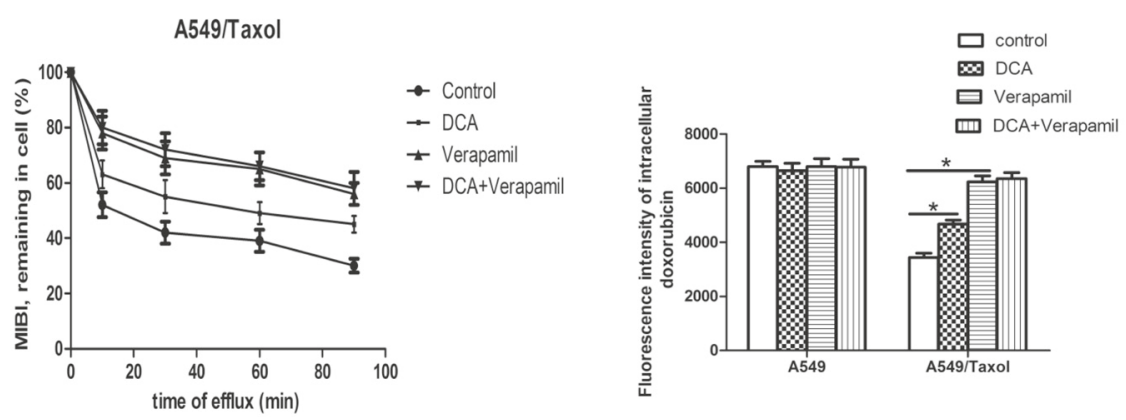

Figure 4 DCA partially inactivates P-glycoprotein activity in drug-resistant cell lines. (A) P-glycoprotein expression in A549 and A549/Taxol cells treated with 0 or $10 \mathrm{mM}$ DCA for $24 \mathrm{~h}$, as determined by western blotting. (B) Influence of DCA, verapamil, or DCA + verapamil on ${ }^{99 \mathrm{~m}} \mathrm{Tc}-\mathrm{MIBI}$ uptake. Verapamil was used as a positive control. (C) Influence of DCA, verapamil, or DCA + verapamilon ${ }^{99 \mathrm{~m}} \mathrm{TC}-\mathrm{MIBl}$ excretion. (D) Influence of DCA, verapamil, or DCA + verapamilon accumulation of doxorubicin. ${ }^{*} P<0.05$. Data are mean \pm SEM of three independent experiments.

In the cytoplasm, citrate can specifically inhibit PFK1, an enzyme critical to glucose metabolism, to reduce glycolysis [40].

We confirmed that citrate levels induced by DCA were significantly different in A549 and A549/Taxol cells (Figure 5B). Citrate was lower in A549/Taxol cells in the absence of DCA; however, $10 \mathrm{mM}$ DCA treatment for $24 \mathrm{~h}$ resulted in an $80 \%$ increase in citrate in A549/ Taxol cells, compared with a $25 \%$ increase in A549 cells (Figure 5B). Citrate accumulation resulted in inhibition of PFK activity (Figure 5C). We examined the effect of DCA on citrate generation under different conditions of mitochondrial damage, and citrate levels declined significantly in hypoxia or A549/MD cell models with mitochondrial respiratory deficiency (Figure 5D). However, citrate levels were higher after DCA treatment compared withA549 cells under normoxia. Other cell lines such as H1299 (p53 deficiency) and HCCLM3 (highly aggressive) [23] can tolerate much higher concentrations of paclitaxel compared withA549 cells, with IC50 concentrations 2.3- and 3.0-fold higher than A549 cells, respectively. Similarly, we observed that citrate levels declined and DCA treatment induced greater citrate accumulation in these cell lines compared with A549 cells.

We next used PDK2 siRNA to inhibit PDK and mimic DCA treatment. PDK2 was chosen because it is the only widely expressed isoenzyme and has the lowest $\mathrm{Ki}$ for DCA [41]. Treatment with PDK2 siRNA inhibited PDK2 expression in A549 and A549/Taxol cells (Figure 5E). Consistent with DCA treatment, knockdown of PDK2 for $72 \mathrm{~h}$ resulted in greater cell death in A549/Taxol cells than in A549 cells. However, PDK2 siRNA cells were not sensitive to DCA treatment (Figure $5 \mathrm{~F}$ ). We confirmed that 
A

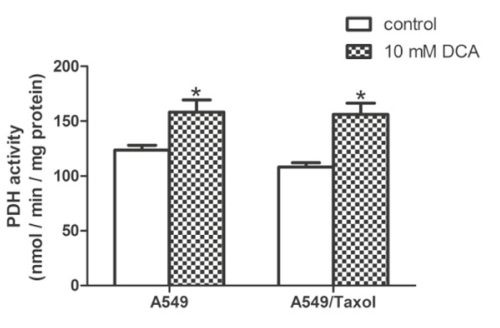

C

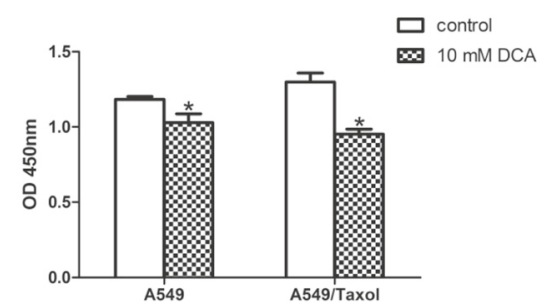

E

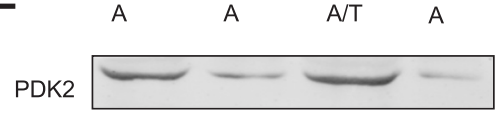

Actin

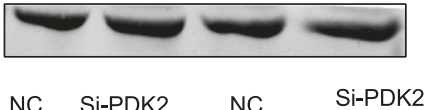

G

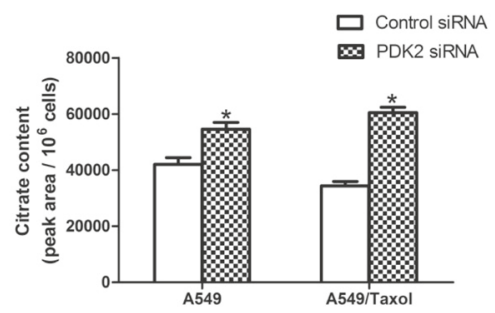

B

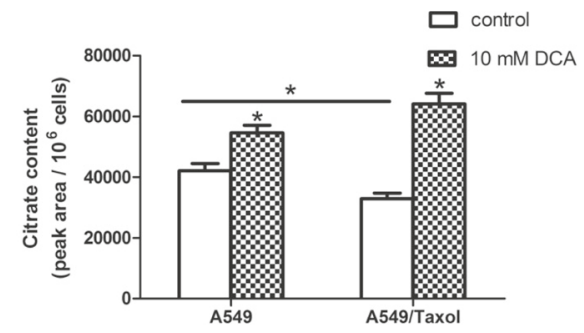

D

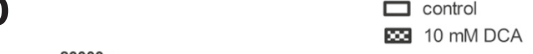

F

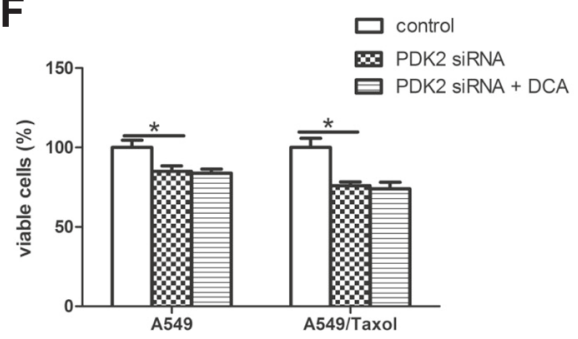

H

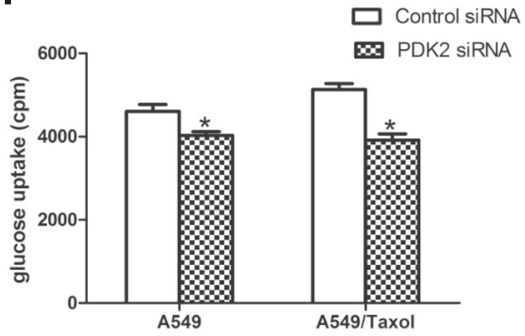

Figure 5 DCA increases citrate accumulation in cells with mitochondrial injury. (A) Effects of a 3-h DCA treatment on PDH activity in A549 and A549/ Taxol cells. (B) Influence of DCA on citrate accumulation measured by GC. (C) Influence of DCA on PFK activity by PFK colorimetric assay. (D) Intracellular citrate levels under normoxia in various cell lines and hypoxic conditions in A549 cell lines treated with 0 or 10 mM DCA for 24 h. (E) Comparative analysis of PDK2 expression in control and PDK2-siRNA cell lines determined by western blotting (48 h post-transfection). (F) Viability of A549 and A549/Taxol cells treated with PDK2 siRNA. (G) Influence of PDK2 siRNA on citrate accumulation. (H) Glucose uptake in control and PDK2-siRNA cells. ${ }^{*} P<0.05$. Data are mean \pm SEM of three independent experiments.

PDK2 siRNA increased citrate and decreased glucose uptake more significantly in A549/Taxol cells, which was consistent with DCA treatment (Figure 5G, H).

\section{DCA improved in vivo efficacy of paclitaxel in A549/Taxol cell xenografts}

Treatment with paclitaxel alone did not significantly suppress tumor volume (Figure 6A) or weight (Figure 6B) compared with the control group. In contrast, a combination of DCA and paclitaxel decreased tumor volume by $78 \%$, compared with a decrease of only $8 \%$ with paclitaxel alone (relative tumor size to vehicle-treated tumors after 3 weeks'treatment; $P<0.001$; Figure 6 A). Similarly, combined treatment decreased tumor weight by $84 \%$ compared with9\% with paclitaxel alone (relative tumor weight to vehicle-treated tumors after 3 weeks'treatment; $P<0.001$; 

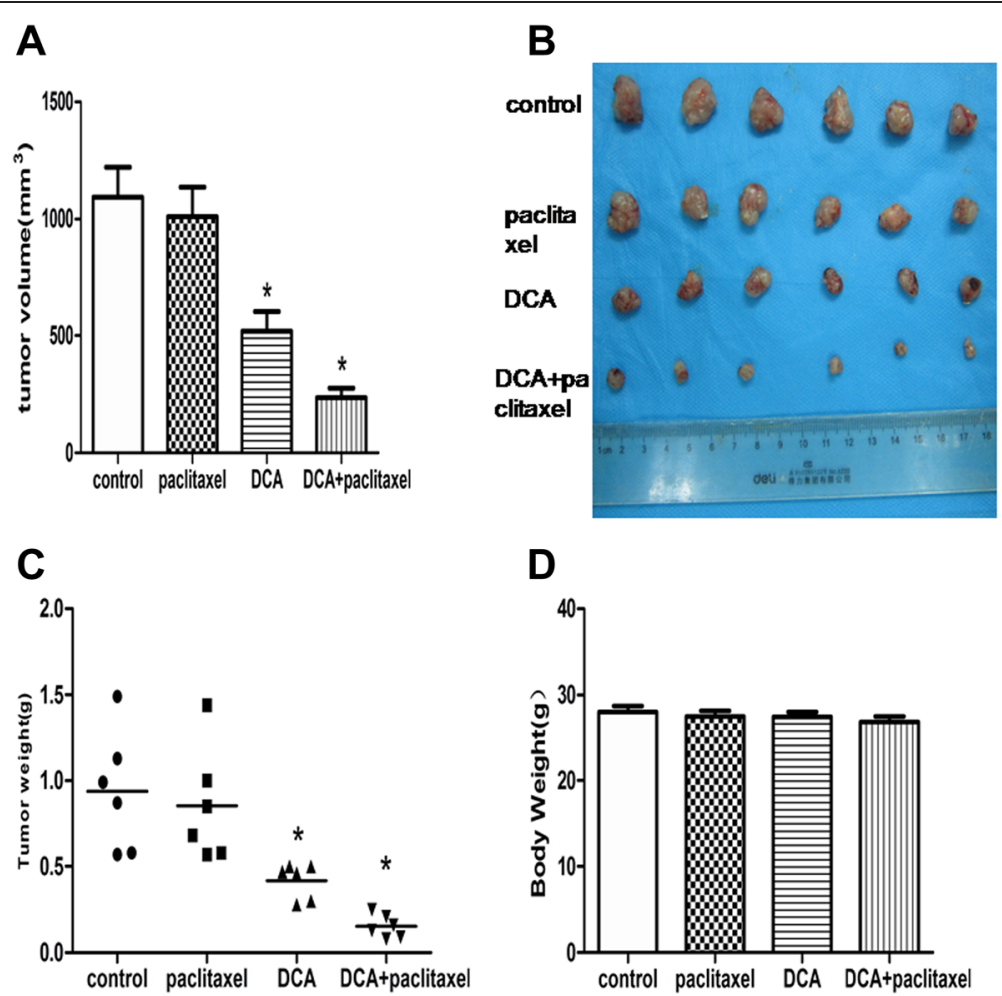

Figure 6 Effect of paclitaxel and DCA alone and in combination on the growth of A549/Taxol xenografts in nude mice. (A-C) In vivo growth of tumors in mice treated with DCA alone or in combination with paclitaxel was significantly inhibitedcompared with control mice, whereas treatment with paclitaxel alone had no effect. (D) Effect of vehicle, paclitaxel, DCA, or combined treatment on body weight. ${ }^{*} P<0.05$. Data are mean \pm SEM of three independent experiments.

Figure $6 \mathrm{~B}, \mathrm{C})$. No apparent body weight loss or toxicity was observed in any treatment group (Figure 6D). These results provide in vivo evidence that DCA restores drug sensitivity in A549/Taxol cells.

\section{Discussion}

In this study, drug resistance to paclitaxel in tumor cells was closely linked with mitochondrial damage, and mitochondrial dysfunction persisted in A549 cells with acquired resistance. A549/MD cells with stable mitochondrial respiratory deficiency exhibited similar paclitaxel resistance. The mechanism by which mitochondrial respiratory defects cause resistance is complicated. Hypoxia-inducible factor 1 can lead to drug resistance through increased glycolysis and down-regulation of Bid and Bax. The damage of electron transport chain complexes could decrease mitochondrial apoptosis response leading to apoptosis resistance [42]. The present study confirmed that P-glycoprotein expression was significantly increased and induced injury to the electron transport chain in A549 cells. P-glycoprotein is an important resistance protein that can prevent apoptosis by excreting paclitaxel [38]. Increased P-glycoprotein expression due to mitochondrial damage is therefore a potential explanation for paclitaxel resistance in lung cancer.
In this study, DCA targeted A549/Taxol cells specifically and reversed paclitaxel resistance. Surprisingly, what sets this study apart from others is how DCA targets cells with mitochondrial respiratory defects, which was not due to its ability to activate oxidative respiration. Rather, DCA inhibited glutamine oxidation significantly between control and DCA treated cells in both cell lines. However, DCA inhibited glutamine oxidation by $34.4 \%$ in A549/Taxol cells and 19.1\% in A549 cells.A549/Taxol cells were affected by DCA's inhibition of glutamine oxidation significantly more than A549 cells (Figure 3C).

Although DCA did not activate oxidative respiration in A549/Taxol cells compared withA549 cells, and inhibited glucose uptake in both cell types, it inhibited glycolysis more effectively in A549/Taxol cells. We did observe DCA reversed paclitaxel resistance by inhibiting glycolysis. Tumor cells rely on ATP to maintain drug resistance, and decreased ATP can lead to decreased drug resistance [43]. DCA clearly decreased ATP generation in A549/Taxol cells, presumably by inhibiting glycolysis, but failed to reduce ATP production in A549 cells that exhibited better mitochondrial function.

Intracellular ATP is mainly produced by glycolysis and oxidative phosphorylation, and analysis of the main cellular energy sources showed that cells with mitochondrial respiratory defects are more dependent on energy from glycolysis. 
DCA significantly inhibited glycolysis and oxidative phosphorylation of glutamine in A549/Taxol cells, and increased oxidative phosphorylation of glucose could not compensate for the lost energy. This would inevitably lead to reduced ATP generation in A549/Taxol cells. P-glycoprotein is important in paclitaxel resistance, and maintenance of $\mathrm{P}$ glycoprotein activity requires a steady supply of ATP [44]. Therefore, DCA may suppress P-glycoprotein activity by inhibiting glycolysis, which could restore paclitaxel susceptibility in resistant cells with mitochondrial respiratory defects.

Citric acid is generated by citrate synthase following DCA activation of PDC [29], and citrate can then enter the TCA cycle, be oxidized in mitochondria, or enter the cytoplasm via dedicated carrier proteins [39]. However, we found that the TCA cycle was not activated in cells with mitochondrial respiratory defects, suggesting accumulation of intermediates from the generation of acetyl-CoA (by activated PDC). Citrate is both a substrate of and intermediate in the TCA cycle, therefore DCA may induce the accumulation of citrate in cells with mitochondrial respiratory defects. In this study, DCA induced greater levels of citrate accumulation (80\%) in resistant A549/Taxol cells than in sensitive A549 cells. Similarly, DCA induced greater accumulation of citrate in other cell types with mitochondrial respiratory defects, including A549/MD cells that are deficient in mitochondrial respiration under hypoxic conditions.

Citrate lies in a central position in the regulation of cell metabolism and participates in the synthesis of fatty acids in the cytoplasm. Citrate inhibits PFK1 to reduce glycolysis and operates as a key regulator, balancing oxidation and glycolysis [39]. We noted that cells with mitochondrial respiratory defects had decreased levels of citrate, which is conducive for maintaining the Warburg effect to balance energy supply and biosynthesis. Citrate may therefore be used as an indicator of mitochondrial respiratory defects and paclitaxel resistance in tumors, and future metabolic studies on tumors should take this into account.

\section{Conclusions}

In summary, paclitaxel-resistant cells exhibited increased mitochondrial respiratory defects and decreased citric acid levels. DCA induced citrate accumulation that inhibited glycolysis and led to inactivation of P-glycoprotein and reduced drug resistance. These results will be useful for future treatment using DCA alone or in combination with other chemotherapeutic drugs, and aid our understanding of the metabolic regulatory mechanisms in tumors.

\section{Additional files}

Additional file 1: Figure S1. A549/MD cells have minimal complex I activity than A549 cells. *indicate significant differences $(p<0.05)$. Data are means \pm SEM of three independent experiments.
Additional file 2: Figure S2. Expression of P-gp was increased in A549/MD cells compared to A549 cells. *indicate significant differences ( $p<0.05)$. Data are means \pm SEM of three independent experiments.

\section{Abbreviations}

3BrPA: 3-bromopyruvate; DCA: Dichloroacetate; DMEM: Dulbecco's modified eagle's medium; ECAR: Extracellular acidification rate;

FDG: Fluorodeoxyglucose; MIBI: Methoxyisobutylisonitrile; OCR: Oxygen consumption rate; PDC: Pyruvate dehydrogenase complex; PDH: Pyruvate dehydrogenase; PDK: Pyruvate dehydrogenase kinase;

PFK1: Phosphofructokinase1; TCA cycle: Tricarboxylic acid cycle.

\section{Competing interests}

The authors declare that they have no competing interests.

\section{Authors' contributions}

HG designed the experiments. ZX, CRH, YZH, LR and LJ performed the experiments. ZX, CRH, ZXP, SSL and LJ performed the statistical analysis. ZX, $\mathrm{CRH}$ and $\mathrm{HG}$ wrote the manuscript. All authors approved the final draft of this manuscript.

\section{Acknowledgements}

This work was supported by grants from the National Natural Science Foundation of China (Nos. 30830038, 30970842, 81071180, 81101073 and 81471708), the Major State Basic Research Development Program of China (973 Program) (No. 2012CB932604), the New Drug Discovery Project (No. 2012ZX09506-001-005) and the Biomedical Engineering Cross Research Fund of Shanghai Jiao Tong University (No.YG2012MS13). We thank Dr. Yaer Hu of Shanghai Jiao Tong University School of Medicine for herassistance in isotopic tracing analysis. Wealso thank Dr. Yumin Liu of Instrumental Analysis Center of Shanghai Jiao Tong University for her assistance in GC/MS analysis of cellular citrate content.

\section{Author details}

'Department of Nuclear Medicine, Ren Ji Hospital, School of Medicine, Shanghai Jiao Tong University, 1630 Dongfang Road, Shanghai 200127, China. ${ }^{2}$ School of biomedical engineering, Shanghai Jiao Tong University, Shanghai, China. ${ }^{3}$ Department of Cancer Metabolism, Institute of Health Sciences, Chinese Academy of Sciences and Shanghai Jiao Tong University School Medicine, Shanghai, China.

Received: 17 July 2014 Accepted: 3 March 2015

Published online: 19 March 2015

\section{References}

1. Warburg O. On the origin of cancer cells. Science. 1956;123:309-14.

2. Cairns RA, Harris IS, Mak TW. Regulation of cancer cell metabolism. Nat Rev Cancer. 2011;11:85-95.

3. Bensinger SJ, Christofk HR. New aspects of the Warburg effect in cancer cell biology. Semin Cell Dev Biol. 2012;23:352-61.

4. Zhou X, Chen R, Xie W, Ni Y, Liu J, Huang G. Relationship between 18 F-FDG accumulation and lactate dehydrogenase A expression in lung adenocarcinomas, the. J Nucl Med. 2014;55:1766-71.

5. Fantin VR, St-Pierre J, Leder P. Attenuation of LDH-A expression uncovers a link between glycolysis, mitochondrial physiology, and tumor maintenance. Cancer Cell. 2006;9:425-34.

6. Zu XL, Guppy M. Cancer metabolism: facts, fantasy, and fiction. Biochem Biophys Res Commun. 2004;313:459-65.

7. Carew JS, Zhou Y, Albitar M, Carew JD, Keating MJ, Huang P. Mitochondrial DNA mutations in primary leukemia cells after chemotherapy: clinical significance and therapeutic implications. Leukemia. 2003;17:1437-47.

8. Michelakis ED, Webster L, Mackey JR. Dichloroacetate (DCA) as a potential metabolic-targeting therapy for cancer. Brit J Cancer. 2008;99:989-94.

9. Stacpoole PW, Nagaraja NV, Hutson AD. Efficacy of dichloroacetate as a lactate-lowering drug. J Clin Pharmacol. 2003;43:683-91.

10. Bonnet S, Archer SL, Allalunis-Turner J, Haromy A, Beaulieu C, Thompson R, et al. A mitochondria-K+ channel axis is suppressed in cancer and its normalization promotes apoptosis and inhibits cancer growth. Cancer Cell. 2007;11:37-51 
11. Stacpoole PW. The pharmacology of dichloroacetate. Metabolism. 1989:38:1124-44

12. Cao WG, Yacoub S, Shiverick KT, Namiki K, Sakai Y, Porvasnik S, et al. Dichloroacetate (DCA) sensitizes both wild-type and over expressing $\mathrm{BCl}-2$ prostate cancer cells in vitro to radiation. Prostate. 2008;68:1223-31.

13. Robey IF, Martin NK. Bicarbonate and dichloroacetate: evaluating pH altering therapies in a mouse model for metastatic breast cancer. BMC Cancer. 2011;11:235

14. Madhok BM, Yeluri S, Perry SL, Hughes TA, Jayne DG. Dichloroacetate induces apoptosis and cell-cycle arrest in colorectal cancer cells. Brit J Cancer. 2010;102:1746-52.

15. Wong JY, Huggins GS, Debidda M, Munshi NC, De Vivo I. Dichloroacetate induces apoptosis in endometrial cancer cells. Gynecol Oncol. 2008;109:394-402.

16. Cairns RA, Bennewith KL, Graves EE, Giaccia AJ, Chang DT, Denko NC. Pharmacologically increased tumor hypoxia Can Be measured by F-18 fluoroazomycin arabinoside positron emission tomography and enhances tumor response to hypoxic cytotoxin PR-104. Clin Cancer Res. 2009;15:7170-4.

17. Heshe D, Hoogestraat S, Brauckmann C, Karst U, Boos J, Lanvers-Kaminsky C. Dichloroacetate metabolically targeted therapy defeats cytotoxicity of standard anticancer drugs. Cancer Chemother Pharmacol. 2011:67:647-55.

18. Stockwin LH, Yu SX, Borgel S, Hancock C, Wolfe TL, Phillips LR, et al. Sodium dichloroacetate selectively targets cells with defects in the mitochondrial ETC. Int J Cancer. 2010;127:2510-9.

19. Tan M, Yu D. Molecular mechanisms of ErbB2-Mediated breast cancer chemoresistance. Breast Cancer Chemosensitivity. 2007;608:119-29.

20. Donnenberg VS, Donnenberg AD. Multiple drug resistance in cancer revisited: the cancer stem cell hypothesis. J Clin Pharmacol. 2005:45:872-7.

21. Stewart DJ. Tumor and host factors that may limit efficacy of chemotherapy in non-small cell and small cell lung cancer. Crit Rev Oncol Hemat. 2010;75:173-234

22. Sun QL, Sha HF, Yang XH, Bao GL, Lu J, Xie YY. Comparative proteomic analysis of paclitaxel sensitive A549 lung adenocarcinoma cell line and its resistant counterpart A549-Taxol. J Cancer Res Clin. 2011;137:521-32.

23. Tian J, Tang ZY, Ye SL, Liu YK, Lin ZY, Chen J, et al. New human hepatocellular carcinoma (HCC) cell line with highly metastatic potential (MHCC97) and its expressions of the factors associated with metastasis. Brit J Cancer. 1999:81:814-21.

24. Li J, Zhao S, Zhou X, Zhang T, Zhao L, Miao P, et al. Inhibition of lipolysis by mercaptoacetate and etomoxir specifically sensitize drug-resistant lung adenocarcinoma cell to paclitaxel. PLoS One. 2013;8:e74623.

25. Xu RH, Pelicano H, Zhou Y, Carew JS, Feng L, Bhalla KN, et al. Inhibition of glycolysis in cancer cells: a novel strategy to overcome drug resistance associated with mitochondrial respiratory defect and hypoxia. Cancer Res. 2005:65:613-21.

26. Zhou QQ, Lam PY, Han D, Cadenas E. Activation of c-Jun-N-terminal kinase and decline of mitochondrial pyruvate dehydrogenase activity during brain aging. FEBS Lett. 2009;583:1132-40.

27. Yu ZH, Zhao XP, Huang LQ, Zhang T, Yang FJ, Xie L, et al. Proviral Insertion in Murine Lymphomas 2 (PIM2) Oncogene Phosphorylates Pyruvate Kinase M2 (PKM2) and promotes glycolysis in cancer cells. J Biol Chem. 2013;288:35406-16.

28. Huang L, Yu Z, Zhang T, Zhao X, Huang G. HSP40 interacts with pyruvate kinase M2 and regulates glycolysis and cell proliferation in tumor cells. PLoS One. 2014;9:e92949.

29. Metallo CM, Gameiro PA, Bell EL, Mattaini KR, Yang JJ, Hiller K, et al. Reductive glutamine metabolism by IDH1 mediates lipogenesis under hypoxia. Nature. 2012;481:380-U166.

30. Waki A, Yano R, Yoshimoto M, Sadato N, Yonekura Y, Fujibayashi Y. Dynamic changes in glucose metabolism accompanying the expression of the neural phenotype after differentiation in PC12 cells. Brain Res. 2001;894:88-94.

31. Lorke DE, Kruger M, Buchert R, Bohuslavizki KH, Clausen M, Schumacher U. In vitro and in vivo tracer characteristics of an established multidrugresistant human colon cancer cell line. J Nucl Med. 2001;42:646-54.

32. Hyafil F, Vergely C, Du Vignaud P, Grand-Perret T. In vitro and in vivo reversal of multidrug resistance by GF120918, an acridonecarboxamide derivative. Cancer Res. 1993:53:4595-602.

33. Dai C, Tiwari AK, Wu CP, Su X, Wang SR, Liu D, et al. Lapatinib (Tykerb, GW572016) reverses multidrug resistance in cancer cells by inhibiting the activity of ATP-binding cassette subfamily B member 1 and G member 2 (vol 68, pg 7905, 2008). Cancer Res. 2008;68:10387.
34. Yu Z, Ge Y, Xie L, Zhang T, Huang L, Zhao X, et al. Using a yeast two-hybrid system to identify FTCD as a new regulator for HIF-1alpha in HepG2 cells. Cell Signal. 2014;26:1560-6.

35. Kim HS, Oh JM, Jin DH, Yang KH, Moon EY. Paclitaxel induces vascular endothelial growth factor expression through reactive oxygen species production. Pharmacology. 2008;81:317-24.

36. Frezza C, Gottlieb E. Mitochondria in cancer: not just innocent bystanders. Semin Cancer Biol. 2009;19:4-11.

37. Fulda S, Galluzzi L, Kroemer G. Targeting mitochondria for cancer therapy. Nat Rev Drug Discov. 2010;9:447-64.

38. Fletcher Jl, Haber M, Henderson MJ, Norris MD. ABC transporters in cancer: more than just drug efflux pumps. Nat Rev Cancer. 2010;10:147-56.

39. Icard P, Poulain L, Lincet $H$. Understanding the central role of citrate in the metabolism of cancer cells. Bba-Rev Cancer. 2012;1825:111-6.

40. Lu Y, Zhang $X$, Zhang H, Lan J, Huang G, Varin E, et al. Citrate induces apoptotic cell death: a promising way to treat gastric carcinoma? Anticancer Res. 2011;31:797-805.

41. Bowker-Kinley MM, Davis WI, Wu PF, Harris RA, Popov KM. Evidence for existence of tissue-specific regulation of the mammalian pyruvate dehydrogenase complex. Biochem J. 1998;329:191-6.

42. Lemarie A, Grimm S. Mitochondrial respiratory chain complexes: apoptosis sensors mutated in cancer. Oncogene. 2011;30:3985-4003.

43. Zhou Y, Tozzi F, Chen J, Fan F, Xia L, Wang J, et al. Intracellular ATP levels are a pivotal determinant of chemoresistance in colon cancer cells. Cancer Res. 2012;72:304-14.

44. Nakano A, Tsuji D, Miki H, Cui Q, El Sayed SM, Ikegame A, et al. Glycolysis inhibition inactivates $A B C$ transporters to restore drug sensitivity in malignant cells. PLoS ONE. 2011;6:e27222.

\section{Submit your next manuscript to BioMed Central and take full advantage of:}

- Convenient online submission

- Thorough peer review

- No space constraints or color figure charges

- Immediate publication on acceptance

- Inclusion in PubMed, CAS, Scopus and Google Scholar

- Research which is freely available for redistribution 